\title{
Autophagy Induction by $\alpha$-Santalol in Human Prostate Cancer Cells
}

\author{
COLE WALTERS*, MAVERICK REED*, SAMANTHA BARTHOLOMEW* and AJAY BOMMAREDDY \\ Department of Pharmaceutical Sciences, Nesbitt School of Pharmacy, \\ Wilkes University, Wilkes-Barre, PA, U.S.A.
}

\begin{abstract}
Background/Aim: Previous studies have shown that the sandalwood oil constituent $\alpha$-santalol inhibits growth of cultured human prostate cancer cells in vitro and PC-3 prostate cancer xenografts. Along with the studies from our laboratory, it is well established that $\alpha$-santalol targets the phosphatidylinositol-4,5-bisphosphate 3-kinase-AKT serinel threonine kinase 1 (AKT) pathway to induce apoptosis but its growth-suppressive effects have not been fully elucidated. The current study was undertaken to investigate the role of autophagy in $\alpha$-santalol-induced prostate cancer cell death. Materials and Methods: Cell lines LNCaP and PC-3 were maintained in an atmosphere of 95\% air and 5\% CO2 at $37^{\circ} \mathrm{C}$. Trypan blue dye exclusion assay was employed to assess the effects of $\alpha$-santalol with/without 3-methyl adenine on the cell viability of prostate cancer cells. Acidic vesicular organelles induced by $\alpha$-santalol treatment were detected by staining with acridine orange. Immunofluorescence and immunoblotting were performed to analyze expression of proteins involved in the AKT-mammalian target of rapamycin (mTOR) pathway. Results: LNCAP and PC-3 cells upon treatment with $\alpha$-santalol resulted in characteristic features analogous to autophagic response, including formation of acidic vesicular organelles, recruitment and cleavage of microtubule-associated protein 1 light chain 3 (LC3) to autophagosomes. Alpha-santalol treatment further suppressed phosphorylation of activated AKT and mTOR, which are critical regulators of autophagic response. In addition, pretreatment of PC-3 cells with specific inhibitor of autophagy
\end{abstract}

This article is freely accessible online.

*These Authors contributed equally to this study.

Correspondence to: Ajay Bommareddy, Ph.D., Department of Pharmaceutical Sciences, Nesbitt School of Pharmacy, Wilkes University, 84 W. South Street, Wilkes-Barre, PA 18766, U.S.A. Tel: +1 5704084220, Fax: +1 5704084299, e-mail: ajay.bommareddy@wilkes.edu

Key Words: Prostate cancer, autophagy, alpha-santalol, autophagosomes. (3-methyladenine) and co-treatment with $\alpha$-santalol attenuated the expression of LC3-II and phospho-AKT, and significantly reduced the cell viability. Conclusion: The present study indicates that $\alpha$-santalol induces autophagy by targeting the AKT-mTOR pathway in prostate cancer cells, which may serve as a protective mechanism.

Due to a high mortality of men with prostate cancer in the United States, strategies to lessen or eliminate this deadly malignancy are in high demand. Previous studies suggest that oil from sandalwood (Santalum album) and its components, including $\alpha$-santalol (Figure 1), offer protection against the development of various chronic ailments including prostate cancer $(1,2)$. For example, studies employing non-melanoma and melanoma skin cancer cells, upon treatment with $\alpha$ santalol showed growth suppression through cell-cycle arrest/apoptosis induction $(3,4)$ and exhibited microtubule depolymerization in UACC-62 melanoma cells similar to that by vinblastine (3). Not only was the ability of $\alpha$-santalol to prevent chemically-induced cancer in various experimental animal models well established (1), but accumulating evidence also suggests its potential to induce growth suppression of prostate cancer cells by inducing apoptosis $(5,6)$. Administration of $\alpha$-santalol resulted in inhibition of tumor growth and tumor angiogenesis in a PC-3 xenograft tumor model through suppression of the AKT serine/threonine kinase 1 (AKT)-mammalian target of rapamycin (mTOR)-p70S6K pathway (7). Despite these findings in prostate tumor models, the antitumor effects of $\alpha$-santalol against human prostate cancer growth are not fully understood.

The PI3K/AKT signal transduction pathway is well known for its major role in cellular metabolism, survival, proliferation, tumor development, growth and metastasis (8). mTOR, a protein downstream in the phosphatidylinositol4,5-bisphosphate 3-kinase/AKT signaling pathway, aids in cell growth regulation and metabolism, however, when deregulated, mTOR causes excessive cell proliferation, thus leading to the development of unwanted growth. mTOR inhibition leads to induction of autophagy. Autophagy, a well-defined evolutionarily conserved catabolic process that 


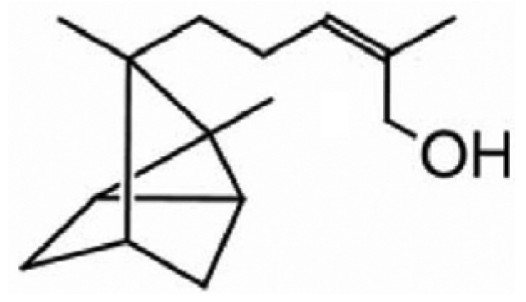

Figure 1. Structure of $\alpha$-santalol.

involves lysosomal degradation of cellular material, provides energy and macromolecular precursors for cellular functions (9, 10). Autophagy, which typically starts with the development of a phagophore, proceeds with recruitment and processing of microtubule-associated protein 1 light chain 3 (LC3)-I to LC3-II occurring in the presence of an autophagic stimulus, with ultimate autophagosomal sequestration. Eventually, the freshly developed phagophore engulfs cellular proteins and organelles, and fuses with a lysosome, forming an autophagolysosome, which performs autophagy (10). Based on the stimuli and tumor type, autophagy can be neutral, tumor-promoting, or tumor-suppressive (11). Autophagy can serve as a protective mechanism in the beginning stages of cancer cell growth and can also favor growth of pre-existing tumors (12). Owing to the multifaceted role of autophagy, it is critical to identify its association in the growth and development of cancer. Studies from our laboratory and published reports identified that $\alpha$ santalol targets PI3K/AKT pathway to inhibit prostate cancer cell growth, tumor growth and angiogenesis in PC-3 tumor xenografts (5-7). Therefore, we were interested in exploring the role of autophagy in $\alpha$-santalol-induced cell death of human prostate cancer cells. We demonstrate for the first time that $\alpha$-santalol-induced autophagy in prostate cancer cells may serve as a defensive mechanism and that its therapeutic efficacy can be enhanced in the presence of a known autophagy inhibitor.

\section{Materials and Methods}

Reagents. Extraction of $\alpha$-santalol used in the current study was described by us in our previous publication (5). All the tissue culture consumables and cell-culture reagents, including RPMI 1640 medium, F-12K medium, fetal bovine serum, and penicillin/ streptomycin antibiotic mixture were purchased from InvitrogenLife Technologies (Carlsbad, CA, USA). Total AKT, p-AKT, LC-3, mTOR, p-mTOR, p62 antibodies used in the current study were purchased from Cell Signaling (Danvers, MA, USA), actin from Sigma-Aldrich (St. Louis, MO, USA) and all other essential chemicals and reagents were purchased in their highest purity available.
Cell lines. Human prostate cancer cell lines LNCaP and PC-3, which were authenticated previously by the Research Animal Diagnostic Laboratory (University of Missouri, Columbia, MO, USA), were a generous gift from Dr. Shivendra V. Singh (University of Pittsburgh Cancer Institute, Pittsburgh, USA). Both cell lines as described in our previous studies $(5,6)$ were maintained in their respective growth culture media in an atmosphere of $95 \%$ air and $5 \% \mathrm{CO}_{2}$ at $37^{\circ} \mathrm{C}$. Each experiment was carried out with a freshly prepared stock solution of $\alpha$ santalol in dimethyl sulfoxide (DMSO) and adding an equal volume of DMSO (final concentration $<0.5 \%$ ) to the control-treated cells.

Cell viability assay. Trypan blue dye exclusion assay was employed to assess the effects of $\alpha$-santalol with/without 3-methyl adenine (3$\mathrm{MA})$ on the cell viability of PC- 3 cells. Briefly, $5 \times 10^{4}$ cells in $1 \mathrm{ml}$ of complete medium were plated in 12-well plates and allowed to attach overnight. After attachment, cells were pre-treated for $2 \mathrm{~h}$ with 3-MA then co-treated with $\alpha$-santalol $(40 \mu \mathrm{M})$ or DMSO for $24 \mathrm{~h}$ at $37^{\circ} \mathrm{C}$. At the end of the incubation, cells (both floating and adherent) were collected and pelleted by centrifuging at 4,500 $\times g$ for 5 minutes. The pellet was resuspended in $30 \mu$ of $0.5 \%$ trypan blue solution in phosphate-buffered saline (PBS), and live (white) cells were counted using a hemocytometer under an inverted microscope as described by us previously $(5,6)$. Cell viability was expressed as a percentage of that of the DMSO-treated control.

Immunoblotting. PC-3 and $\mathrm{LNCaP}$ cells $\left(7.5 \times 10^{5}\right.$ cells for each cell line) after attachment were treated with different concentrations of $\alpha$ santalol (20 and $40 \mu \mathrm{M})$ or DMSO for different time periods (6 or 9 h). After collecting the cells (both floating and attached to the plate), cells were lysed using the protocol described in earlier studies $(5,6)$. Lysate proteins were resolved by sodium-dodecyl sulfate polyacrylamide gel electrophoresis and transferred onto polyvinylidene fluoride membrane. Immunoblotting protocol was carried out as described previously $(5,6)$ and the immunoreactive bands were visualized employing LI-COR-Odyssey infra-red scanner (LI-COR, Lincoln, NE, USA) with actin as a loading control.

Detection of acidic vesicular organelles (AVOs). This experiment was performed by plating PC-3 and LNCaP cells $\left(2 \times 10^{5}\right)$ on coverslips in a 12 -well plate and allowing attachment by overnight incubation. After treating the cells with DMSO (control) or $\alpha$ santalol for $24 \mathrm{~h}$, cells were subjected to staining with acridine orange in PBS for 15 minutes. After incubation, acridine orange stain was removed and the cells were washed with PBS. Coverslips were mounted on glass slides and then the cells were examined under a microscope with fluorescence attached with Lumenera Infinity 3 camera at high power magnification for AVOs as described previously (13).

Statistical analysis. All experiments were performed a minimum of two times with triplicate measurements for quantitative comparisons. Student's $t$-test or a one-way analysis of variance was completed to determine if there was a statistical significance of differences in measured variables between control and treated groups. It was then followed by Bonferroni's multiple comparison test.

\section{Results}

Autophagy induction in prostate cancer cells, as supported by acridine orange staining. An important characteristic of 
A

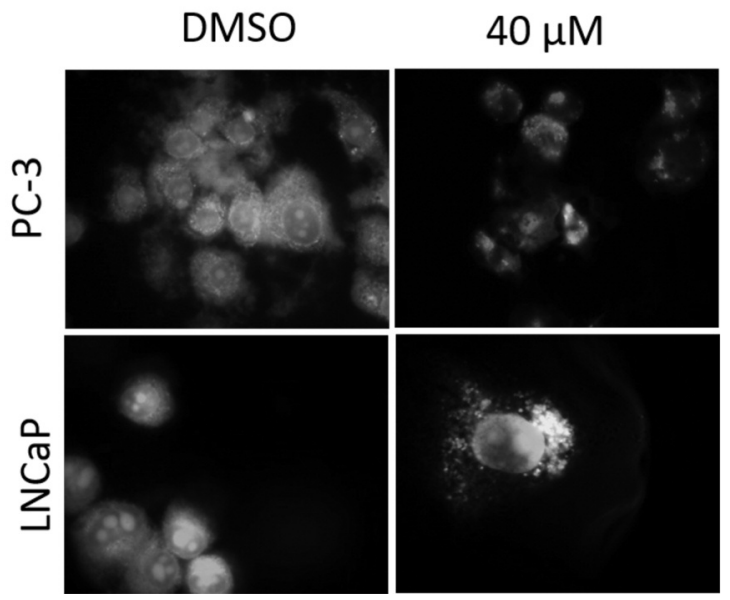

B

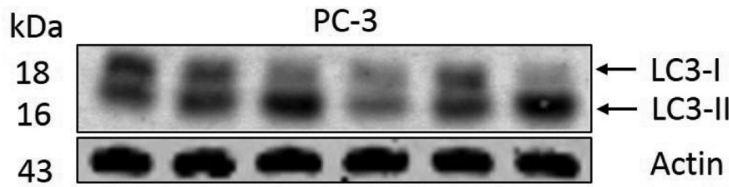

LNCaP

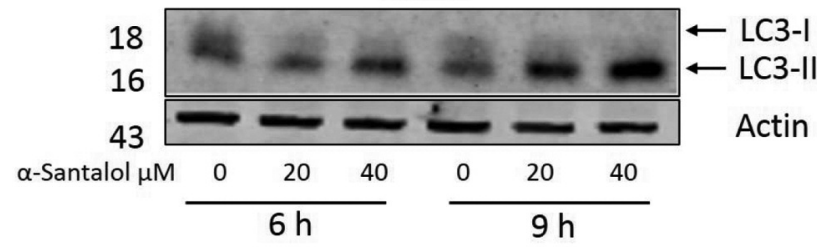

Figure 2. A: Representative acridine orange staining images $(\times 100)$ of LNCaP and PC-3 cells treated with dimethyl sulfoxide (DMSO) or 40 umol/l of $\alpha$-santalol for 9 h. B: Immunoblotting for LC3 and actin levels using lysates from $\mathrm{LNCaP}$ and $P C-3$ cells.

cells engaged in autophagy involves the formation of AVOs following treatment with different stimuli capable of inducing autophagy. Hence, we decided to investigate the effect of $\alpha$-santalol on the formation of AVOs in LNCaP and PC-3 cells employing fluorescence microscopy after performing the acridine orange staining protocol. Figure $2 \mathrm{~A}$ depicts the effect of $\alpha$-santalol on the formation of AVOs in LNCaP and PC-3 cells. As can be seen in the figure, the DMSO-treated control LNCaP and PC-3 cells exhibited primarily green fluorescence whereas treatment of the cells with $40 \mu \mathrm{M} \alpha$-santalol resulted in the formation of yelloworange AVOs. These results provide evidence that $\alpha$-santalol treatment induced the formation of AVOs (and thus autophagy) in prostate cancer cells.

Effects of $\alpha$-santalol on expression of LC3 in PC3 and LNCaP cell lines. Autophagic response to $\alpha$-santalol

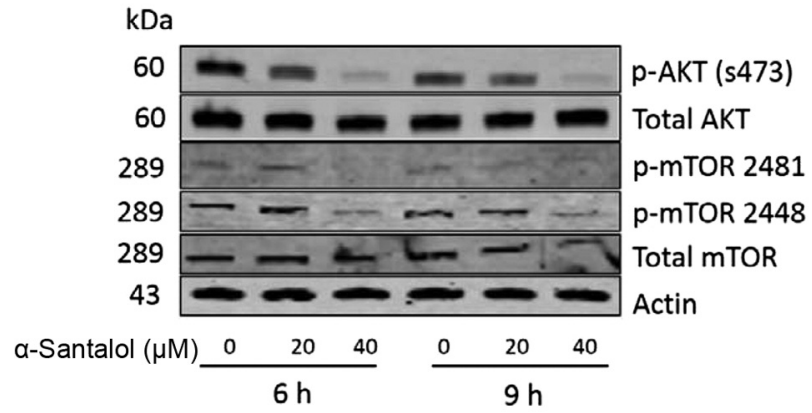

Figure 3. Representative immunoreactive bands of major proteins (phospho-(S473)-AKT serinelthreonine kinase 1 (AKT), total AKT, phospho ( $p$ )-(S2481)-mammalian target of rapamycin (mTOR), $p$ (S2448)-mTOR, total MTOR and actin) in the AKT-mTOR signaling axis using lysates from $\mathrm{PC}-3$ cells.

treatment was further confirmed by examining the processing and recruitment of LC3, an important hallmark of autophagy (14). Expression of LC3-II in cells after exposure to DMSO control, $20 \mu \mathrm{M}$ or $40 \mu \mathrm{M}$ treatment with $\alpha$-santalol for 6 and 9 hours was determined by performing immunoblotting analysis. As presented in Figure 2B, treatment of $\mathrm{LNCaP}$ and PC-3 cells resulted in cleavage of LC3. Expression of cleaved LC3-II was more prominent in the groups treated with $\alpha$-santalol compared to the control group. The expression of cleaved LC3-II was more intense in the group treated with $40 \mu \mathrm{M} \alpha$-santalol compared to that treated with $20 \mu \mathrm{M}$, indicating that autophagic response may be a doseand time-dependent effect. Collectively, the results presented in Figure 2 indicate that $\alpha$-santalol caused autophagy in prostate cancer cells.

$\alpha$-Santalol-induced autophagy correlated with suppression of phosphorylation sites of AKT and mTOR. The protein kinase mTOR, controlled by AKT-mediated phosphorylation has been identified as a negative regulator of autophagy. To understand the mechanism of $\alpha$-santalol-mediated autophagy in our study, we decided to examine the role of $\alpha$-santalol treatment on the expression levels of activated phosphorylation of AKT (S473) and mTOR (S2448, S2481) using PC-3 cells. As shown in Figure 3, $\alpha$-santalol (20 $\mu \mathrm{M}$, and $40 \mu \mathrm{M}$ ) treatment for 6 and 9 hours reduced the phosphorylation of AKT and mTOR. The expression of total AKT and mTOR was only modestly reduced when compared to the control cells. In summary, these results indicated that $\alpha$-santalol-mediated autophagy was regulated by the AKT/mTOR pathway.

Autophagy served as a protective mechanism against $\alpha$ santalol-induced cell death. Autophagic response has been identified to serve as a protective mechanism against 
A
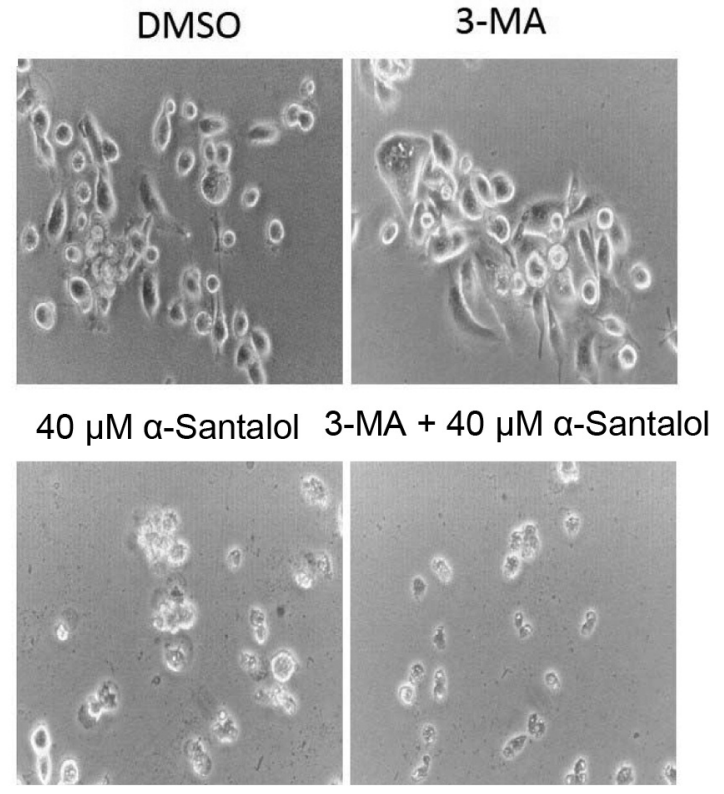

B

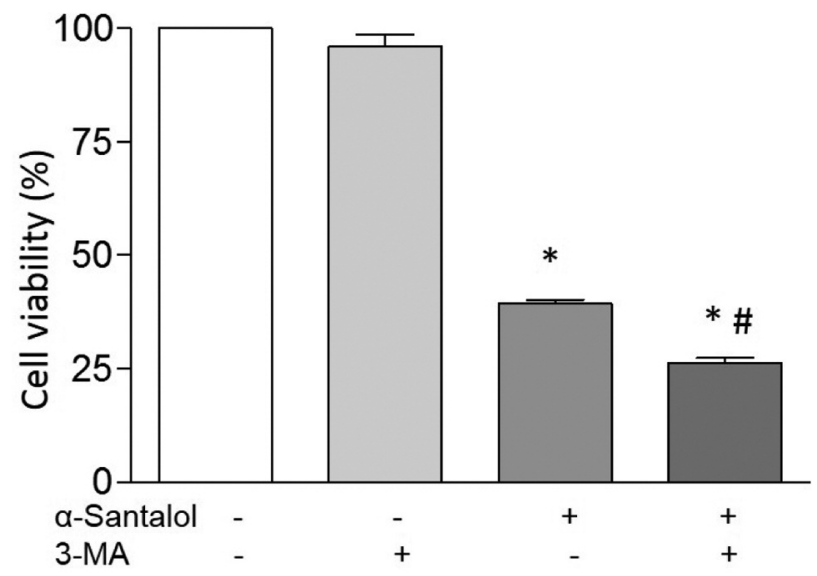

Figure 4. A: Bright field micrographs $(\times 10)$ depicting the morphology of PC-3 cells following 24-h treatment with $40 \mu$ mol/l $\alpha$-santalol with/without 2-h pretreatment with 5 mmol/l 3-methyladenine (3-MA). B: Trypan blue dye-exclusion assay depicting the percentage of cell viability of PC-3 cells following 24-h treatment with $40 \mu \mathrm{mol} / \mathrm{l} \alpha-$ santalol with/without 2-h pretreatment with $5 \mathrm{mmol} / \mathrm{l} 3-\mathrm{MA}$. Data are the means \pm standard error $(n=3)$. Significantly different at $p<0.05$ from *control; ${ }^{*} \alpha$-santalol alone.

apoptotic stimuli as well as starvation conditions. At the same time, autophagy was also suggested to contribute to cell death caused by various anticancer agents, typically referred as type II cell death, distinct from apoptosis (type I cell death). We sought to explore the contribution of $\alpha$ santalol-induced autophagy in growth suppression by

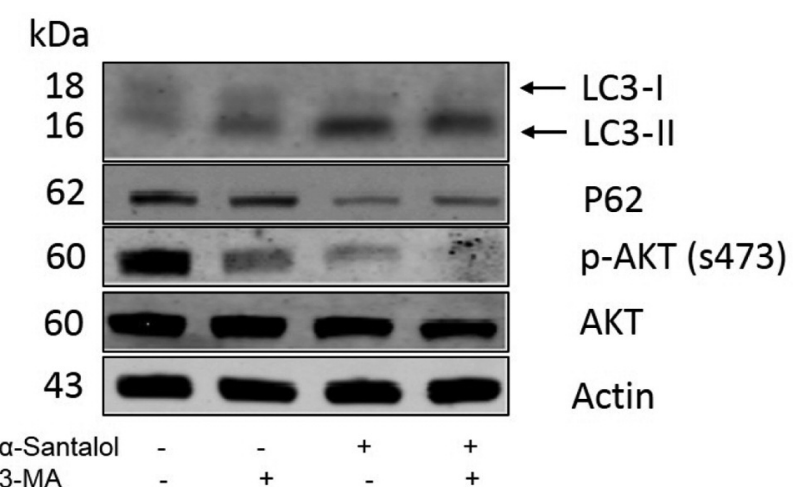

Figure 5. Immunoblotting for microtubule-associated protein 1 light chain 3 (LC3), P62, phospho (p)-(S473)-AKT serine/threonine kinase 1 $(A K T)$, total $A K T$, and actin using lysates from PC-3 cells following 24-h treatment with $40 \mu \mathrm{mol} / \mathrm{l} \alpha$-santalol in the presence and absence of 2-h pretreatment with $5 \mathrm{mmol} / \mathrm{l} 3-\mathrm{MA}$. Comparable results were observed in two independent experiments.

employing a known autophagy inhibitor, 3-MA. Cell viability assay was performed to assess the contribution of autophagy in growth suppression of PC-3 cells. Morphology of cells treated with DMSO, 3-MA, and $\alpha$-santalol, alone and in combination is presented in Figure 4A. As expected, cells in the control and 3-MA-treated group exhibited morphological characteristics similar to those of healthy growing cells, whereas the groups treated with $\alpha$-santalol, alone and with 3-MA, exhibited features distinct from those of DMSO-treated cells. In addition, co-treatment with $\alpha$ santalol and 3-MA statistically significantly reduced cell viability when compared to cells treated with $\alpha$-santalol alone, as shown in Figure 4B. Data from the cell viability assay indicated that inhibition of the autophagic response contributed by $\alpha$-santalol resulted in increased overall cell death of PC-3 cells.

To further confirm the results, we performed immunoblotting analysis of cleaved LC3, P62, and phosphorylation of AKT (S473). As presented in Figure 5, expression levels of these proteins decreased in cells co-treated with 3-MA and $\alpha$-santalol compared with that treated with $\alpha$-santalol alone. It is noteworthy that expression of p-AKT (S473), which is a key regulator of mTOR, was completely abrogated in the cells cotreated with $\alpha$-santalol and 3-MA.

\section{Discussion}

In our first published study employing prostate cancer cells and $\alpha$-santalol, we showed that $\alpha$-santalol induced apoptotic cell death by activating both intrinsic and extrinsic pathways (5). A follow-up study published recently revealed the involvement of PI3K-AKT-survivin pathway in $\alpha$-santalol- 
mediated prostate cancer cell death (6). In the same study, we also showed that $\alpha$-santalol-induced apoptotic cell death increased in the presence of a known inhibitor of the PI3KAKT pathway, in addition to reduced expression of survivin, a downstream effector of AKT. In a PC-3 xenograft tumor model, administration of $\alpha$-santalol resulted in inhibition of tumor growth and tumor angiogenesis through suppression of the AKT-mTOR 70S6K pathway (7). The current study revealed that upon treatment with $\alpha$-santalol, human prostate cancer cells survive through autophagy induction. The combination of AVO detection and conversion of LC3-I to LC3-II provides evidence for autophagy induction in prostate cancer cells treated with $\alpha$-santalol. Our data also support the claim that autophagic response mediated by $\alpha$-santalol may be regulated through inhibition of the AKT-mTOR pathway.

Through acridine orange staining, the visualization of yellowish-orange stained cells treated with $40 \mu \mathrm{M} \alpha$-santalol is indicative of autophagosome formation, due to the stain's $\mathrm{pH}$ sensitive ability to fluoresce when in an acidic environment (i.e. in the low $\mathrm{pH}$ environment of autophagolysosomes and their precursors which markedly increase during autophagy). Unfortunately, acridine orange staining does not explain how the process of autophagy occurs mechanistically within the cell, as there are multiple (potential) mechanisms through which autophagy can be promoted or inhibited. In fact, acridine orange fluoresces in any low $\mathrm{pH}$ environment, so this result is merely indicative of increased autophagy; a low $\mathrm{pH}$ alone does not guarantee the presence of autophagolysosomes. For this reason, we turned to investigating intracellular mediators more specific to autophagolysosome formation: LC3-I and LC3-II. LC3-I is a cytosolic microtubule-associated protein, which when autophagy occurs is converted to LC3-II. Subsequently, LC3-II can be recruited to the membranes of autophagosomes, which in turn fuse with lysosomes to form the autophagolysosomes (15). Our immunoblotting data confirmed the processing of LC3-I to LC3-II in cells treated with $\alpha$-santalol.

The molecular mechanism for autophagy is regulated through the AKT-mTOR signaling axis. AKT has multiple properties which down-regulate pro-apoptotic proteins, and one major function is the activation of mTOR. The mTOR protein has several pro-survival actions overriding apoptosis and autophagy (16). The results from the current study show that $\alpha$-santalol treatment effectively suppressed the AKTmTOR signaling axis. Increasing concentrations of $\alpha$ santalol proportionately reduced expression of the phosphorylated (active) forms of both AKT and mTOR. Interestingly, the total amounts (active and inactive) of AKT and mTOR did not appear to change. Subsequent studies are required to examine the direct role of $\alpha$-santalol in the downregulation of AKT and mTOR phosphorylation. Because the role of autophagy in the context of cancer-relevant stimuli is not fully understood, the results of the present study provide insights into the mechanistic details associated with the effects of $\alpha$-santalol against prostate cancer development. Studies pertinent to agents known to induce autophagy as a cytoprotective mechanism when used in combination with established inhibitors of autophagy significantly increased cell death. For example, pharmacological inhibition of autophagy by 3-MA, bafilomycin and chloroquine augmented apoptotic cell death induced by sulforaphane in different cancer models (17-19). Conversely, autophagyinduced by other agents is not cytoprotective and contributes to overall cellular death $(13,20)$. In the present study, the percentage of cell viability was statistically significantly lower in PC-3 cells co-treated with $\alpha$-santalol and 3-MA compared to those to treated with $\alpha$-santalol alone. The results clearly indicate $\alpha$-santalol-induced autophagy has a cytoprotective mechanism which, when inhibited by 3-MA, caused a significant reduction in cell viability. In an attempt to further understand this interaction between $\alpha$-santalol and 3-MA in PC-3 cells, we performed immunoblotting analysis of phospho-AKT, LC3-II, and P62. As expected, the level of phospho-AKT appeared to be lower in the lysates of cells cotreated with $\alpha$-santalol and 3-MA compared with $\alpha$-santalol alone treatment. Similarly, the immunoreactive band of cleaved LC3-II was slightly lesser in cells co-treated with $\alpha$ santalol and 3-MA. P62, an intracellular protein targeting cytosolic polyubiquitinated molecules for autophagy, is a substrate for autophagic degradation. Therefore, the degradation of P62 can be used as a marker of autophagic clearance (21). In the current study, the expression level of P62 was lower in cells treated with $\alpha$-santalol alone compared to cells co-treated with 3-MA, which further confirm the findings of the study.

While further studies are required to fully elucidate the antitumor properties of $\alpha$-santalol, the following conclusions can be drawn from the findings of the present study: (i) There appears to be increased antitumor effects when cotreating PC-3 cells with $\alpha$-santalol and 3-MA, despite their opposing effects on autophagy; (ii) and perhaps more importantly, this suggests that the induction of autophagy by $\alpha$-santalol is not entirely antitumorigenic. Otherwise, the addition of 3-MA to $\alpha$-santalol-treated cells would permit more cancer cell survival relative to PC-3 cells treated with $\alpha$-santalol alone. Therefore, $\alpha$-santalol might increase autophagy through multiple pro-autophagic interactions (possibly both within and outside of the AKT-mTOR axis), where some of these autophagy-inducing interactions inhibit prostate cancer cell survival but others promote it. Within the realm of prospective treatments for any type of human tumorigenesis, including prostate cancer, the ideal autophagy-inducing treatment will only promote autophagy, which ultimately leads to growth inhibition and death of tumor cells. Likewise, autophagy-inducing agents with desirable antitumor properties would not increase the likelihood of cancer cell survival. 


\section{Conflicts of Interest}

The Authors do not have anything to declare and have no known competing financial interests or personal relationships that could have appeared to influence the work reported in this article.

\section{Authors' Contributions}

A. Bommareddy was responsible for the overall experimental design and supervision of the experiments, data analysis and preparation of the final article. C. Walters, M. Reed, and S. Bartholomew helped with article preparation, along with the trypan blue, immunofluorescence and immunoblotting experiments.

\section{Acknowledgements}

This investigation was supported by Wilkes University Provost research grant and mentoring grant awarded to $\mathrm{AB}$ by Wilkes University.

\section{References}

1 Santha S and Dwivedi C: Anticancer effects of sandalwood (Santalum album). Anticancer Res 35: 3137-3145, 2015. PMID: 26026073.

2 Bommareddy A, Brozena S, Steigerwalt J, Landis T, Hughes S, Mabry E, Knopp A, VanWert AL and Dwivedi C: Medicinal properties of alpha-santalol, a constituent of sandalwood oil. Nat Prod Res 33(4): 527-543, 2019. PMID: 29130352. DOI: 10.1080/14786419.2017.1399387

3 Kaur M, Agarwal C, Singh RP, Guan X, Dwivedi C and Agarwal R: Skin cancer chemopreventive agent, $\{$ alpha $\}$-santalol, induces apoptotic death of human epidermoid carcinoma A431 cells via caspase activation together with dissipation of mitochondrial membrane potential and cytochrome c release. Carcinogenesis 26(2): 369-380, 2005. PMID: 15528219. DOI: 10.1093/carcin/ bgh 325

4 Zhang X, Chen W, Guillermo R, Chandrasekher G, Kaushik RS, Young A, Fahmy $\mathrm{H}$ and Dwivedi C: Alpha-santalol, a chemopreventive agent against skin cancer, causes $\mathrm{G} 2 / \mathrm{M}$ cell cycle arrest in both p53-mutated human epidermoid carcinoma A431 cells and p53 wild-type human melanoma UACC-62 cells. BMC Res Notes 3: 220, 2010. PMID: 20682067. DOI: 10.1186/1756-0500-3-220

5 Bommareddy A, Rule B, VanWert AL, Santha S and Dwivedi C: $\alpha$-Santalol, a derivative of sandalwood oil, induces apoptosis in human prostate cancer cells by causing caspase- 3 activation. Phytomedicine 19: 804-811, 2012. PMID: 22571975. DOI: 10.1016/j.phymed.2012.04.003

6 Bommareddy A, McGlynn D, Lewis M, Lockus L, Seward J, Hong KL, VanWert AL and Dwivedi C. AKT/surviving pathway inhibition enhances apoptotic cell death-induced by $\alpha$-santalol in human prostate cancer cells. Fitoterapia 143: 104552, 2020. PMID: 32173422. DOI: 10.1016/j.fitote.2020.104552

7 Saraswati S, Kumar S and Alhaider AA: $\alpha$-Santalol inhibits angiogenesis and growth of human prostate tumor growth by targeting vascular endothelial growth factor receptor 2-mediated AKT/mTOR/P70S6K signaling pathway. Mol Cancer 12: 147, 2013. PMID: 24261856. DOI: 10.1186/1476-4598-12-147
8 Song G, Ouyang G and Bao S: The activation of AKT/PKB signaling pathway and cell survival. J Cell Mol Med 9(1): 59-71, 2005. PMID: 15784165. DOI: 10.1111/j.1582-4934.2005.tb00337.x

9 Yorimitsu T and Klionsky DJ: Autophagy: Molecular machinery for self-eating. Cell Death Differ 12: 1542-1552, 2005. PMID: 16247502. DOI: $10.1038 /$ sj.cdd. 4401765

10 Mulcahy Levy JM, Towers CG and Thorburn A: Targeting autophagy in cancer. Nat Rev Cancer 17: 528-542, 2017. PMID: 28751651. DOI: $10.1038 / \mathrm{nrc} .2017 .53$

11 Amaravadi R, Kimmelman AC and White E: Recent insights into the function of autophagy in cancer. Genes Dev 30: 19131930, 2016. PMID: 27664235. DOI: 10.1101/gad.287524.116

12 Parejo S, Tschan MP, Muraro MG, Garattini E, Spagnoli GC and Schlafi AM: Assessing autophagy during retinoid treatment of breast cancer cells. In: Retinoid and Retinoid Signaling. S.K. Ray (Ed.), Humana, New York, NY. pp. 237-256, 2019.

13 Bommareddy A, Hahm ER, Xiao D, Powolny AA, Fisher A, Jiang $\mathrm{Y}$ and Singh SV: ATG5 Regulates phenethyl isothiocyanateinduced autophagic and apoptotic cell death in human prostate cancer cells. Cancer Res 69(8): 3704-12, 2009. PMID: 19336571. DOI: 10.1158/0008-5472.CAN-08-4344

14 Karna P, Zughaier S, Pannu V, Simmons R, Narayan S and Aneja $\mathrm{R}$ : Induction of reactive species mediated by a novel microtubulemodulating agent. J Biol Chem 285(24): 18737-48, 2010. PMID: 20404319. DOI: 10.1074/jbc.M109.091694

15 Tanida I, Ueno T and Kominami E: LC3 and autophagy. Methods Mol Biol 445: 77-88, 2008. PMID: 18425443. DOI: 10.1007/978-1-59745-157-4_4

16 Porta C, Paglino C and Mosca A: Targeting PI3K/AKT/mTOR signaling in cancer. Front Oncol 4: 64, 2014. PMID: 24782981. DOI: $10.3389 /$ fonc. 2014.00064

17 Nishikawa T, Tsuno NH, Okaji Y, Shuno Y, Sasaki K, Hongo K, Sunami E, Kitayama J, Takahashi K and Nagawa H: Inhibition of autophagy potentiates sulforaphane-induced apoptosis in human colon cancer cells. Ann Surg Oncol 17: 592-602, 2010. PMID: 19830499. DOI: 10.1245/s10434-009-0696-x

18 Kanematsu S, Uehara N, Miki H, Yoshizawa K, Kawanaka A, Yuri $\mathrm{T}$ and Tsubura A: Autophagy inhibition enhances sulforaphane-induced apoptosis in human breast cancer cells. Anticancer Res 30: 3381-3390, 2010. PMID: 20944112.

19 Vyas AR, Hahm ER, Arlotti JA, Watkins S, Stolz DB, Desai D, Amin S and Singh SV: Chemoprevention of prostate cancer by $\mathrm{D}, \mathrm{L}$-sulforaphane is augmented by pharmacological inhibition of autophagy. Cancer Res 73(19): 5985-5995, 2013. PMID: 23921360. DOI: 10.1158/0008-5472.CAN-13-0755

20 Powolny AA, Bommareddy A, Hahm ER, Normolle DP, Beumer JH, Nelson JB and Singh SV: Chemopreventive potential of the cruciferous vegetable constituent phenethyl isothiocyanate in a mouse model of prostate cancer. JNCI 103(7): 571-584, 2011 PMID: 21330634. DOI: 10.1093/jnci/djr029

21 Lippai M and Lów P: The role of the selective adaptor P62 and ubiquitin-like proteins in autophagy. BioMed Res Int 832704: 111, 2014. PMID: 25013806. DOI: 10.1155/2014/832704

Received December 23, 2020

Revised January 21, 2021 Accepted January 25, 2021 